\title{
GENETIC RELATIONSHIP AMONG Musa balbisiana ACCESSIONS AND IDENTIFICATION OF SRAP MARKERS LINKED TO Musa B GENOME \\ Youssef, M. ${ }^{* 1}$ and R. M. Escobedo-GraciaMedrano ${ }^{2}$ \\ 1-Genetics Department, Faculty of Agriculture, Assiut University, Assiut 71526, Egypt \\ 2-Unidad de Bioquímica y Biología Molecular de Plantas, Centro de Investigación Científica de Yucatán, A.C., Calle 43, \# 130, Col. Chuburná de Hidalgo, CP. 97200 \\ Mérida, Yucatán, México \\ * Corresponding author: mkhirshy@aun.edu.eg
}

\begin{abstract}
The sequence related amplified polymorphism (SRAP) was used to assess genetic relationship among some Musa balbisiana related accessions including wild diploid, triploid cultivar, plantains, diploid and tetraploid hybrids. A total of 74 bands were generated, of which 65 bands (87.84\%) were polymorphic among the tested accessions. The average of polymorphism information content (PIC) for all primers was 0.29 , while the average of diversity index (DI) was 0.73 . Results showed the SRAP efficiency in grouping the tested accessions and exposing minimum differences among genotypes. Interestingly, the wild diploid accessions were separated into two groups, in which BB-CICY, Tani, Cameron and Singapuri grouped together with the triploid cultivar "Lep Chang Kut", while the other diploid accessions, i.e. Butuhan, Pisang Batu and BB-545, formed the second group near to plantains and hybrids. These findings were subsequently supported by the identification of some specific bands generated by SRAP, which were existed in all B genome contained accessions with the exception of Butuhan, Pisang Batu and BB-545. The results suggested that these accessions may have recombinant chromosomes of A and B genomes or they are mislabeled. The identification of the specific bands for B genome found in this study provided markers linked to chromosomes 2, 8 and 11, which could be helpful in discriminating banana and plantain cultivars. Specific primers were designed from sequences of some specific bands, among which a 256bp fragment was successfully amplified, and was able to distinguish between acuminata and balbisiana accessions. Moreover, the information herein could be important in Musa basic breeding program, identification of mislabeled accessions and germplasm management and conservation.
\end{abstract}

Keywords: Musa, banana, SRAP, sequencing, specific markers, B genome

\section{INTRODUCTION}

Banana and plantain are belong to the genus Musa (family Musaceae), which was recently divided into two main sections, the first is Musa, which includes all species related to pre-classified Eumusa and Rhodochlamys, and the second section is Callimusa that includes species of the two other sections (Australimusa and Callimusa) (Häkkinen 2013). The world production of banana and plantain reaches approximately 105.96 and 37.88 million tons in 2013, respectively and are produced by about 120 countries (FAOSTAT 2015), which reflects their great importance worldwide. Musa balbisiana Colla is one progenitor of cultivated banana which contains B genome with a basic chromosome number of $2 \mathrm{n}=22$ (Davey et al. 2013). M. balbisiana is important for banana breeding program due to its valuable agronomic traits including vigor, resistance to pest, disease and drought (Ahmad et al. 2014). M. balbisiana and $M$. acuminata (A genome) formed plantains $(\mathrm{AAB})$ and cooking banana $(\mathrm{ABB})$, diploid (AB) and tetraploid hybrid (AAAB, AABB, ABBB) (Teo et al. 2005). Unlike the well understood $M$. acuminata, only few genetic diversity studies of $M$. balbisiana have been conducted (Ahmad et al. 2014). In addition, there is no record of subspecies classification of M. balbisiana (Ude et al. 2002).

Several molecular markers have been used previously with Musa spp., to assess genetic diversity and reveal relationship among Musa species and subspecies, including random amplified polymorphic DNA, RAPD (Ferreira et al. 2004), amplified fragment length polymorphism, AFLP (El-Khishin et al. 2009), inter simple sequence repeats, ISSR (Poerba et al.
2010), simple sequence repeats, SSR (Hippolyte et al. 2012), diversity array technology, DArT (Risterucci et al. 2009), restriction fragment length polymorphism, RFLP (Nwakanma et al. 2003), Variable number tandem repeat, VNTR (Crouch et al. 1999) and sequence related amplified polymorphism, SRAP (Youssef et al. 2011). Among these markers, SRAP has been confirmed to be more efficient and informative than other since it targets the open reading frames $(\mathrm{Li}$ and Quieros 2001). SRAP showed its excellency in exposing high level of polymorphism, several specific bands, clear relationship among Musa sections and high degree of differentiation between A and B genomes, as well as within plantains and cooking banana (Phothipan et al. 2005; Youssef et al. 2011; Valdez-Ojeda et al. 2014; Pinar et al. 2015). In addition, Youssef et al. (2011) indicated that SRAP was able to generate several unique and specific bands for M. balbisiana accessions which were existed in various genome compositions i.e. $\mathrm{BB}, \mathrm{AB}, \mathrm{AAB}, \mathrm{ABB}, \mathrm{AABB}$ and $\mathrm{AAAB}$. These bands are of great importance to discriminate accessions contain B genome from other genotypes and successively can help in Musa breeding programs, germplasm management, as well as rapid identification of mislabeled accessions.

The present study is an extension to the previous work of Youssef et al. (2011) with more focus on $M$. balbisiana and B genome. Thus, in this study the number of $M$. balbisiana wild accessions was increased to be analyzed together with different accessions having $\mathrm{B}$ genome and to confirm the presence of the specific bands generated previously by SRAP. Identification of these bands by analysis of their sequences could assist 
in focusing on the genome regions containing such markers especially after Musa genome sequence became publically available (D'Hont et al. 2012). Therefore, the present study aimed to assess the genetic relationship among $M$. balbisiana related accessions using SRAP markers and to identify the B specific bands sequences.

\section{MATERIALS AND METHODS}

\section{Plant material}

Thirteen accessions were used in this study, consisting of wild diploid of Musa balbisiana, triploid cultivar, plantains, diploid and tetraploid hybrids, as well as a double haploid $M$. acuminata as presented in Table 1. Young cigar leaves were collected from plants of the Musa germplasm collection of the Centro de Investigación Científica de Yucatán (CICY) held at the research station of the Instituto Nacional de Investigaciones Forestales Agrícolas y Pecuarias (INIFAP) at Uxmal, Yucatán, México (20² 24' 40.10" Lat. N, and $89^{\circ} 45^{\prime} 24.90^{\prime \prime}$ Long.W, 8.0 meters above sea level, m.a.s.l). All Musa accessions with an International Transit Code (ITC) number were provided by the Biodiversity International Centre for Musa, located at the Laboratory for Tropical Plant Improvement in the Catholic University of Leuven (Belgium), whereas accessions BB-CICY and MalCICY were collected at Teapa (Tabasco) in México.

Table 1. Accessions used in the present study.

\begin{tabular}{|c|c|c|c|c|c|c|}
\hline No. & Species/hybrid & $\begin{array}{c}\text { Subspecies/ } \\
\text { subgroup }\end{array}$ & Genome & Name & Abbreviation & ITC $^{b}$ \\
\hline 1 & M. acuminata & malaccensis & AA & CIRAD 930 & CRD & 1511 \\
\hline 2 & M. balbisiana & - & $\mathrm{BB}$ & Tani & TAN & 1120 \\
\hline 3 & M. balbisiana & - & $\mathrm{BB}$ & Cameron & CAM & 0246 \\
\hline 4 & M. balbisiana & - & $\mathrm{BB}$ & Butuhan & BUT & 0565 \\
\hline 5 & M. balbisiana & - & $\mathrm{BB}$ & Balbisiana & B545 & 0545 \\
\hline 6 & M. balbisiana & - & $\mathrm{BB}$ & Balbisiana CICY & BB-CICY & $*$ \\
\hline 7 & M. balbisiana & - & $\mathrm{BB}$ & Singapuri & SIN & 0248 \\
\hline 8 & M. balbisiana & - & $\mathrm{BB}$ & Pisang batu & PBT & 1156 \\
\hline 9 & $M . a \times M . b$ & Ney Poovan & $\mathrm{AB}$ & Kunnan & KUN & 1034 \\
\hline 10 & M. balbisiana & Lep Chang Kut & $\mathrm{BBB}$ & Lep Chang Kut & LCK & 0647 \\
\hline 11 & Tetraploid hybrids & - & $\mathrm{AAAB}$ & FHIA-01 & FHI & 0504 \\
\hline 12 & Plantains & Pome & $\mathrm{AAB}$ & Prata ana & PRA & 0962 \\
\hline 13 & Plantains & Plantain & $\mathrm{AAB}$ & Orishele & ORI & 0517 \\
\hline $14^{\mathrm{a}}$ & M. acuminata & burmanicoides & AA & Calcuta-IV & CAL & 0249 \\
\hline $15^{\mathrm{a}}$ & M. acuminata & malaccensis & AA & Malaccensis-399 & Mal-399 & 0399 \\
\hline $16^{\mathrm{a}}$ & M. acuminata & malaccensis & $\mathrm{AA}$ & Malaccensis-250 & Mal-250 & 0250 \\
\hline $17^{\mathrm{a}}$ & M. acuminata & malaccensis & AA & Malaccensis-CICY & Mal-CICY & $*$ \\
\hline
\end{tabular}

${ }^{\mathrm{a}}$ Accessions used for confirmation of specific bands, ${ }^{\mathrm{b}}$ International Transit Code, * Accessions from Teapa, Tabasco, Mexico.

\section{DNA Extraction and SRAP amplification}

Total genomic DNA from Musa accessions was extracted from $100 \mathrm{mg}$ of frozen young cigar leaves following the protocol of Youssef et al. (2015). DNA quality and concentration were determined using a spectrophotometer according to Stulnig and Amberger (1994). The SRAP protocol was implemented using the tools of Khirshyat 1.0 (Youssef 2012). Five primer combinations were selected after Youssef et al. (2011) based on generation of specific bands for Musa accessions containing B genome as shown in Table 2. Each $20 \mu \mathrm{l}$ SRAP amplification reaction consisted of 2 $\mu \mathrm{l}$ of $10 \times$ PCR buffer, $1.6 \mu \mathrm{l}$ of $50 \mathrm{mM} \mathrm{MgCl} 2,1.6 \mu \mathrm{l}$ of $10 \mu \mathrm{M}$ of each forward and reverse primer, $2.5 \mu \mathrm{l}$ of $2 \mathrm{mM}$ dNTPs, $25 \mathrm{ng}$ template DNA and $0.25 \mu \mathrm{l}$ of $5 \mathrm{U}$ Taq-DNA polymerase (Invitrogen). The PCR was carried out with the initial cycle at $94^{\circ} \mathrm{C}$ for $2 \mathrm{~min}, 5$ cycles of $94^{\circ} \mathrm{C}$ for $30 \mathrm{~s}, 35^{\circ} \mathrm{C}$ for $30 \mathrm{~s}$ and $72^{\circ} \mathrm{C}$ for 1 min, another 35 cycles of $94^{\circ} \mathrm{C}$ for $30 \mathrm{~s}, 50^{\circ} \mathrm{C}$ for $30 \mathrm{~s}$ and $72^{\circ} \mathrm{C}$ for $1 \mathrm{~min}$, and the final extension at $72^{\circ} \mathrm{C}$ for 5 min. SRAP amplification products were visualized using ethidium bromide in $3 \%$ agarose gel.

\section{Identification of specific bands for $B$ genome}

Four accessions were used for the recovery of specific bands i.e. BB-CICY, Singapuri, Tani and Prata ana. SRAP amplification products of these samples were separated on $6 \%$ polyacrylamide gel to insure clear separation. Specific bands for B genome were recovered from the gel and purified using ethanol precipitation. For each fragment, four samples were sequenced, each in forward and reverse sense at Macrogen, Inc. using a standard Sanger sequencing method. Sequences were automatically aligned using ClustalW in Sequencher 5.2.3 (Gene Codes) after removing the low-quality end regions (Q score <20). Subsequently, the Sequencher alignment file was exported in the FASTA format. Sequences were analyzed against NCBI database and Banana-Hub. Only important sequences according to the alignment of Banana-Hub were used for specific primer design (Table 2). For confirmation, four additional diploid accessions (AA) of $M$. acuminata ssp. malaccensis and ssp. burmanicoides were used (Table 1). PCR was performed for the amplification of the specific bands as $94^{\circ} \mathrm{C}$ for $2 \mathrm{~min}$ as initial denaturation followed by 30 cycles of $94^{\circ} \mathrm{C}$ for $1 \mathrm{~min}, 55^{\circ} \mathrm{C}$ for $1 \mathrm{~min}$ and $72^{\circ} \mathrm{C}$ for $1 \mathrm{~min}$, and a final extension at $72^{\circ} \mathrm{C}$ for $3 \mathrm{~min}$. PCR products were visualized on $2 \%$ agarose using ethidium bromide. 
Table 2. SRAP primer sequences used in the present study.

\begin{tabular}{llll}
\hline No. & Code & Forward primer $\left(\mathbf{5}^{\prime}-\mathbf{3}^{\prime}\right)$ & Reverse primers $\left(\mathbf{5}^{\prime} \mathbf{- 3}^{\prime}\right)$ \\
\hline 1 & Me1-Em1 & GACTGCGTACGAATTAAT & TGAGTCCAAACCGGATA \\
2 & Me2-Em1 & GACTGCGTACGAATTAAT & TGAGTCCAAACCGGAGC \\
3 & Me4-Em1 & GACTGCGTACGAATTAAT & TGAGTCCAAACCGGACC \\
4 & Me3-Em8 & GACTGCGTACGAATTGAC & TGAGTCCAAACCGGTGT \\
5 & Me4-Em8 & GACTGCGTACGAATTAGC & TGAGTCCAAACCGGACC \\
$6^{*}$ & BB-246bp & ACATACCAGGGTTTACCGG & CGTCATTAGGGATACGTACG \\
$7^{*}$ & BB-256bp & TTGGGCTAAGGTACAACGG & TGGTTAGGCATGACTCAGC \\
${ }^{*}$ Specific primers designed for specific bands amplification.
\end{tabular}

\section{Molecular data analysis}

The presence (1) or the absence (0) of bands was made in a binary matrix from SRAP profiles. The percentage of polymorphism $(\% \mathrm{P})$ was calculated by dividing the number of polymorphic bands with the total number of generated bands. The polymorphism information content (PIC) was estimated for each marker using $\mathrm{PIC} i=2 f_{i}\left(1-f_{i}\right)$, where $\mathrm{PIC} i$ is the $\mathrm{PIC}$ of the marker $i, f_{i}$ is the frequency of the amplified allele (band present), and (1-fi) is the frequency of the band absent (Roldan-Ruiz et al. 2000). The resolving power $(\mathrm{R} p)$ of each primer in all markers was calculated using

the formula: , where

, and $p$ is the proportion of the accessions containing the I band (Prevost and Wilkinson, 1999). Diversity index (DI) was calculated using Nei's (1987) equation $D I=\frac{n\left(1-\Sigma\left[p_{0}^{2}\right]\right)}{n-1}$, individuals analyzed and $p_{i}$, $\mathrm{n}$ is the number of individuals anatyzed allele. The software NTSYSpc ver. 2.20s was used and genetic similarities were computed using Jaccard's coefficient (1908). Cluster analysis was carried out on similarity estimates using UPGMA. Statistical stability of the branches in the dendrogram was tested by bootstrap analysis with 1,000 replicates using the Free Tree 0.9.1.50 software program (Hampl et al. 2001).

\section{RESULTS AND DISCUSSION}

Assessment of genetic diversity and relationship helps in exposing the relatedness of genotypes and facilities the selection of useful genotypes in breeding and improvement programs. Most of the previous studies on Musa genetic diversity mainly focused on the diversity of $M$. acuminata and its related cultivars. However, the study of genetic variation in M. balbisiana and its hybrids is only in the beginning (Valdez-Ojeda et al. 2014).

In the present study, different accessions related to $M$. balbisiana were used to assess genetic relationship among B genome contained accessions and to identify some sequences of SRAP bands associated with B genome. SRAP primers were amplified successfully with all tested accessions (Fig. 1). The total number of generated bands was 74, of which 65 bands $(87.84 \%)$ were polymorphic among the tested accessions. The number of amplified bands per primer ranged from 13 to 19 with an average of 14.8 bands. The highest percentage of polymorphism (\%) was
$100 \%$ generated by Me1-Em1, while the lowest was $71.43 \%$ generated by Me4-Em1 as presented in Table 3 . The high genetic variability found in this study within M. balbisiana accessions was in agreement with previous reports using AFLP markers (Ude et al. 2002; Wang et al. 2006) and the morphological variability (Shepherd 1988; Hari 1989; Sotto and Rabara 2000). In addition, polymorphism information content (PIC) was calculated for each primer in the present study. The averaged PIC for all primers was 0.29 and ranged from 0.20 to 0.37 for Me4-Em1 and Me4-Em8, respectively. Moreover, the averaged diversity index (DI) among the tested accessions was 0.73 and ranged from 0.50 to 0.83 for Me4-Em1 and Me1-Em1, respectively. The resolving power $(\mathrm{R} p)$ was calculated as well for each primer, where Me4-Em8 showed the highest $\mathrm{R} p$ (11.08) while Me4-Em1 showed the lowest $\mathrm{Rp}$ (3.85) with an average of 6.52 for all primers as shown in Table 3.

Genetic relationship among $M$. balbisiana related accessions

SRAP showed its efficiency in revealing the genetic relationship among $M$. balbisiana related accessions. Cluster analysis based on UPGMA and Jaccard's coefficient was performed, the dendrogram showed a clear relationship among the tested accessions as presented in figure 2 . In this regard, M. acuminata accession (CIRAD) was out of group and placed in a separated branch at similarity value of 0.36 . While the other 12 accessions, which contain B genome, were divided into two main clusters. Interestingly SRAP separated the diploid accessions (BB) into two groups, the first main cluster gathered BB-CICY, Tani, Cameron and Singapuri with the triploid cultivar (BBB) "Lep Chang Kut" indicating their clear relatedness. While the other diploid accessions (i.e. Butuhan, Pisang batu and BB-545) were separated in the second main cluster (sub-cluster II-A) and assembled near to subcluster II-B, which contained acuminata $\times$ balbisiana hybrids (i.e. Kunnan, FHIA-01, Prata ana and Orishele). These findings are in agreement with the previous studies of Youssef et al. (2011) and Valdez-Ojeda et al. (2014). However, unlike our findings Pisang Batu and BB-CICY have been clustered together using fluorescent SRAP markers (Valdez-Ojeda et al. 2014). In addition, Pisang Batu has a parthenocarpic fruit and is a relative weak plant susceptible to Sigatoka, while BB-CICY is a fertile seedy and vigorous plant; these morphological differences between these accessions supported our findings that they are very divergent. 


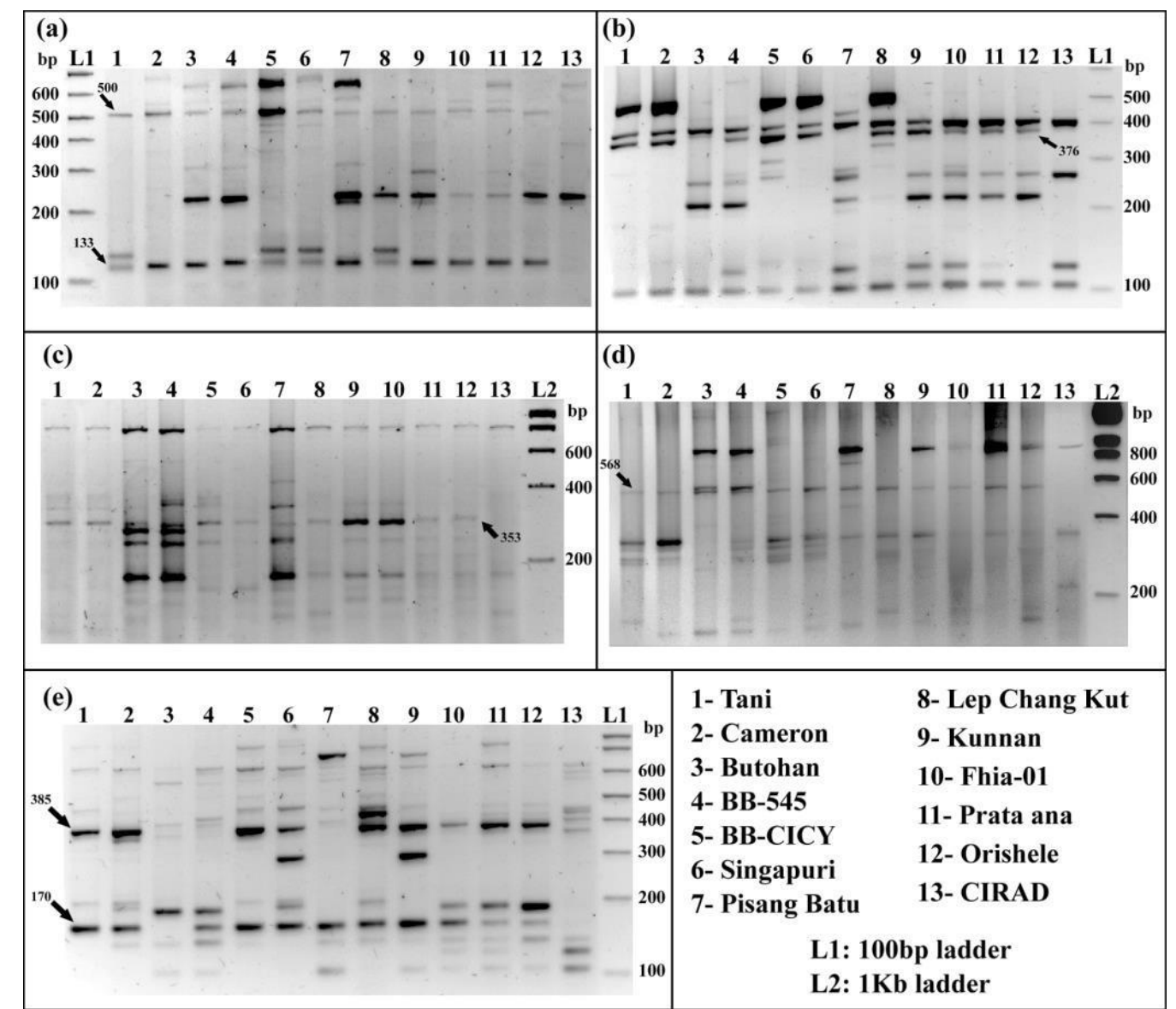

Figure 1. SRAP profiles generated by primers: (a) Me1-Em, (b) Me2-Em1, (c) Me4-Em1, (d) Me8-Em3 and (d) Me4-Em8. Arrows show specific bands for B genome contained accessions.

Table 3. Survey of the percentage of polymorphism, polymorphism information content, primer resolving power and diversity index of the used SRAP primers.

\begin{tabular}{lcccccc}
\hline Primer & TNB & NPB & \%P & PIC & R $\boldsymbol{~}$ & DI \\
\hline Me1-Em1 & 13 & 13 & 100.00 & 0.34 & 6.31 & 0.83 \\
Me2-Em1 & 14 & 12 & 85.71 & 0.30 & 6.15 & 0.75 \\
Me4-Em1 & 14 & 10 & 71.43 & 0.20 & 3.85 & 0.50 \\
Me8-Em3 & 14 & 13 & 92.86 & 0.26 & 5.23 & 0.79 \\
Me4-Em8 & 19 & 17 & 89.47 & 0.37 & 11.08 & 0.77 \\
Total & 74 & 65 & 87.84 & 0.29 & 6.52 & 0.73 \\
\hline
\end{tabular}

TNB: total number of bands, NPB: number of polymorphic bands, \%P: percentage of polymorphism, PIC: polymorphism information content, $\mathrm{R} p$ : resolving power, DI: diversity index.

Genetic similarities (GS) among the tested accessions were calculated using Jaccard's coefficient as shown in Table 4. The M. acuminata accession "CIRAD" showed low GS (0.24 - 0.37) with most of the tested accessions; however it showed moderate GS (0.43) with both BB-545 and Prata ana (Table 4). Within M. balbisiana diploid accessions, the highest GS (0.69) was between Tani and both Cameron and BBCICY, while the lowest (0.33) was between Tani and Butuhan. Additionally, the highest GS showed by the triploid cultivar "Lep Chang Kut" was 0.65 with both Tani and BB-CICY, while it showed lower GS (0.36) with Butuhan followed by 0.44 with Pisang batu. The diploid hybrid AB "Kunnan" used in this study showed highest GS (0.72) with FHIA-01 followed by 0.67 with Prata ana, while it showed lower GS (0.49) with BBCICY. In addition, the two plantain accessions (Prata ana and Orishele) showed the highest GS (0.74) within the tested accessions (Table 4).

SRAP specific bands for $B$ genome

The results of this investigation confirmed the previous finding of Youssef et al. (2011), which indicated that the specific bands were amplified with all B genome contained accessions used in the present study with some exceptions. For instance, as it was expected, the accession CIRAD (AA) failed to generate any of the specific bands for B genome. In addition, among the twelve $M$. balbisiana related accessions, three showed absence of some of the specific bands (i.e. Butuhan, BB-545 and Pisang Batu) as shown in Table 5. Regarding these accessions, in a previous study on genetic diversity in a set of Musa accessions using Ecotilling, Pisang Batu possessed a nucleotide polymorphism pattern of AA (acuminata) type form 
(Till et al. 2010). In addition, Pisang Batu was reported as a mislabeled accession (Valdez-Ojeda et al. 2014), that it could be either a triploid plant of genome constitution $\mathrm{AAB}$, or a diploid plant which may possess recombinant chromosomes with genome constitution $\mathrm{A}^{\mathrm{b}} \mathrm{A}$ or $\mathrm{B}^{\mathrm{a}} \mathrm{B}$ ( $\mathrm{B}$ genome alleles superscripts indicate the possession of the $\mathrm{A}$ genome as background, and vice versa). In the present study, both BB-545 and Butuhan showed the same pattern of Pisang Batu in the absence of some specific bands. These accessions might have the same genome constitution of Pisang Batu or they have mutation in the binding sites of specific band generated SRAP primers. On the other hand, the fertile diploid accession BB-CICY was suggested that it probably has a $\mathrm{B}^{\mathrm{a}} \mathrm{B}$ constitution, as hypothesized by backcrossing involving primary $\mathrm{AB}$ or $\mathrm{BA}$ hybrids (Valdez-Ojeda et al. 2014). However, in this study BBCICY was able to amplify all B specific bands and showed high genetic similarity with other diploid (BB) and triploid (BBB) accessions.

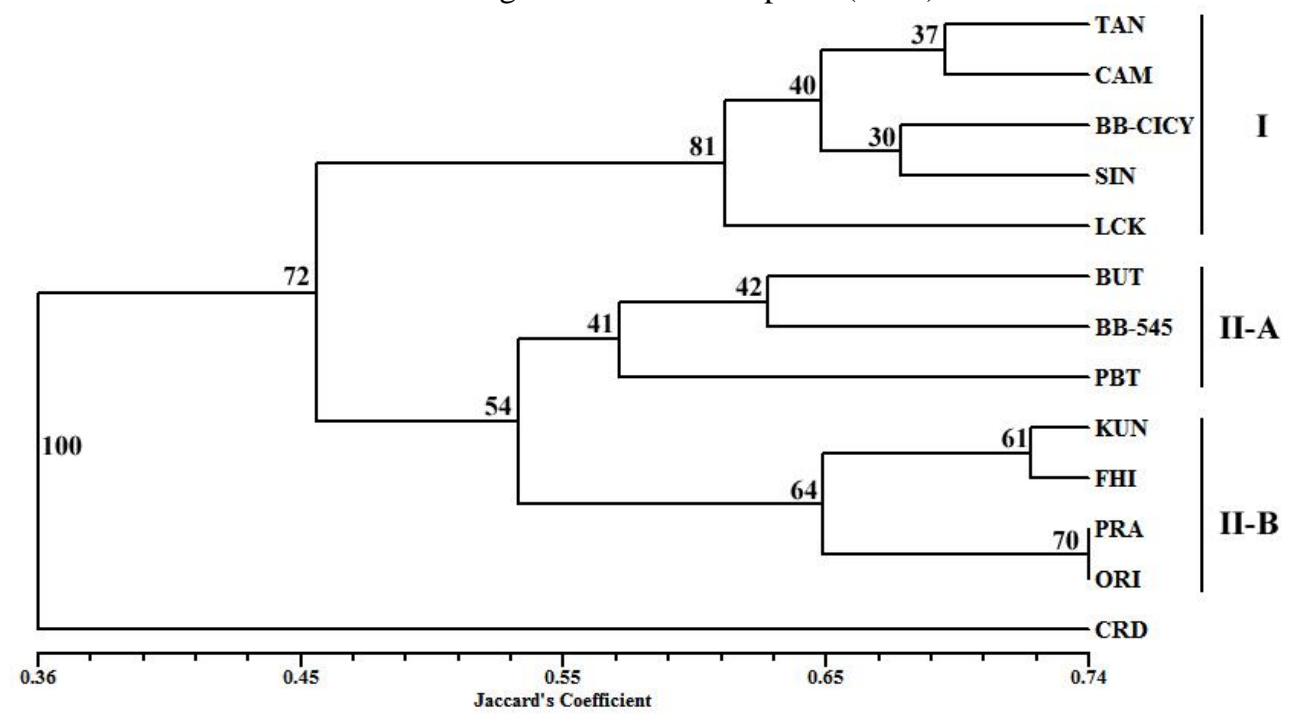

Figure 2. Dendrogram of the tested accessions using UPGMA cluster analysis based on SRAP data with Jaccard's coefficient, numbers over branches indicate the bootstrapping.

Table 4. Genetic similarities among the tested accessions generated by Jaccard's coefficient.

\begin{tabular}{|c|c|c|c|c|c|c|c|c|c|c|c|c|}
\hline 总 & $\underset{k}{Z}$ & $\sum_{u}$ & 定 & 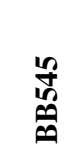 & $\underset{U}{\circlearrowright}$ & 交 & $\underline{a}$ & Uِ & 㝋 & 国 & $\underline{a}$ & 증 \\
\hline CAM & 0.69 & & & & & & & & & & & \\
\hline BUT & 0.33 & 0.41 & & & & & & & & & & \\
\hline B545 & 0.48 & 0.48 & 0.63 & & & & & & & & & \\
\hline BBCICY & 0.69 & 0.61 & 0.38 & 0.49 & & & & & & & & \\
\hline SIN & 0.64 & 0.64 & 0.37 & 0.44 & 0.67 & & & & & & & \\
\hline PBT & 0.38 & 0.34 & 0.52 & 0.62 & 0.40 & 0.35 & & & & & & \\
\hline LCK & 0.65 & 0.57 & 0.36 & 0.54 & 0.65 & 0.57 & 0.44 & & & & & \\
\hline KUN & 0.55 & 0.51 & 0.47 & 0.60 & 0.49 & 0.50 & 0.57 & 0.54 & & & & \\
\hline FHI & 0.51 & 0.44 & 0.47 & 0.58 & 0.43 & 0.43 & 0.61 & 0.51 & 0.72 & & & \\
\hline PRA & 0.46 & 0.46 & 0.51 & 0.55 & 0.44 & 0.48 & 0.51 & 0.55 & 0.67 & 0.68 & & \\
\hline ORI & 0.53 & 0.50 & 0.49 & 0.56 & 0.48 & 0.46 & 0.49 & 0.57 & 0.61 & 0.62 & 0.74 & \\
\hline CRD & 0.24 & 0.31 & 0.41 & 0.43 & 0.30 & 0.27 & 0.41 & 0.33 & 0.36 & 0.41 & 0.43 & 0.37 \\
\hline
\end{tabular}

Table 5. Presence and absence of unique and specific bands among the tested accessions.

\begin{tabular}{|c|c|c|c|c|c|c|c|c|c|c|c|c|c|c|}
\hline Primer & bp & $\underset{E}{Z}$ & $\sum_{\circlearrowright}$ & $\stackrel{\xi}{\ominus}$ & $\begin{array}{l}n \\
10 \\
10 \\
0\end{array}$ & $\underset{\nu}{\circlearrowright}$ & Z & $\underline{n}$ & 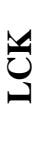 & $\underset{\Xi}{Z}$ & 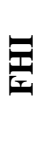 & $\underset{\underline{\alpha}}{\overleftarrow{\alpha}}$ & $\underline{0}$ & $\stackrel{\theta}{\underline{u}}$ \\
\hline Me1-Em1 & 133 & + & + & + & + & + & + & + & + & + & + & + & + & - \\
\hline Me1-Em1 & 500 & + & + & - & - & + & + & - & + & + & + & + & + & - \\
\hline Me2-Em1 & 376 & + & + & - & + & + & + & - & + & + & + & + & + & - \\
\hline M4-Em1 & 353 & + & + & + & + & + & + & + & + & + & + & + & + & - \\
\hline Me8-Em3 & 568 & + & + & + & + & + & + & + & + & + & + & + & + & - \\
\hline Me4-Em8 & 170 & + & + & - & + & + & + & + & + & + & + & + & + & - \\
\hline Me4-Em8 & 385 & + & + & - & - & + & + & - & + & + & + & + & + & - \\
\hline
\end{tabular}


Identification of specific bands for $B$ genome

Out of the seven specific bands, five bands were sequenced and analyzed using sequencher software; those were M1 (133bp by Me1-Em1), M2 (500bp by Me1-Em1), M3 (376bp by Me2-Em1), M4 (170bp by Me4-Em8) and M5 (385bp by Me4-Em8). The consensus sequence of each band was generated from sequences of four accessions (i.e. Tani, Cameron, BB-CICY and Prata ana) after removing the low quality end regions. The software showed that only three sequences were clean and showed a good quality (i.e. M3, M4 and M5), which were used for NCBI and banana-Hub alignment using BLAST tool. Results showed that no significant alignment was found with NCBI; however banana-Hub BLAST tool gave good alignments with the specific sequences. In this regard,
M3 sequence showed $100 \%$ identity (296/296) with Evalue of e-166 when aligned with Pisang Klutuk Wulung (PKW) genome (BB-chromosome 11), while the same sequence showed lower identity (95\%, 282/296) with E-value of e-130 when aligned with double haploid (DH) Pahang genome (AA-chromosome 11) in banana-Hub (Fig. 3). The M4 showed 100\% identity with 8e-81 E-value with PKW genome (BBchromosome 8) and $96 \%$ identity (147/152) with Evalue of $8 \mathrm{e}-69$ with $\mathrm{DH}$-pahang genome (AAchromosome 8) (Fig. 4). In addition, the M5 fragment showed $88 \%$ identity (273/309) and E-value of 2e-83 when aligned with PKW genome (BB-chromosome 2), while it gave $85 \%$ identity (258/302) with E-value of $8 \mathrm{e}-65$ when aligned with DH-pahang genome (AAchromosome 2) (Fig. 5).

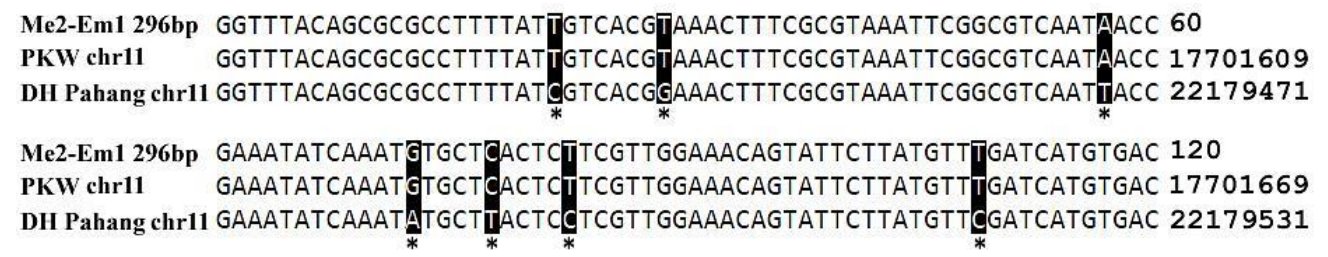

$\begin{array}{ll}\text { Me2-Em1 296bp } & \text { TCCATCTGATGAAGATCATAAAATATACGGTTGGGATTTAACTAAGCACATCGGGAAAGT } 180 \\ \text { PKW chr11 } & \text { TCCATCTGATGAAGATCATAAAATATACGGTTGGGTTTAACTAAGCACATCGGGAAGT } 17701729 \\ \text { DH Pahang chr11 TCCATCTGATCAAGATCATAAAATATACGGTTGGGATTAACTAAGCACATCGGGAAAGT }\end{array}$ $\begin{array}{ll}\text { Me2-Em1 296bp } & \text { AAGTTGGCAAGATTTTCCTATCTTAGCACCTAAAGTACGTACGTATCCCTAATGACGTAT } 240 \\ \text { PKW chr11 } & \text { AAGTTGGCAAGATTTCCTATCTTAGCACCTAAAGTACGTACGTATCCCTAATGACGTAT } 17701789 \\ \text { DH Pahang chr11 AAGTTGC-AAGAGTTCCTACCTTAGCACCTAAATTACGTACGTATCCCTAATGACGTAT } 22179591\end{array}$

$\begin{array}{ll}\text { Me2-Em1 296bp } & \text { TTGAACACAATGCATATCCACATAAATTAATATTTTATTGTGCTCCTATTAATTCG } 296 \\ \text { PKW chr11 } & \text { TTGAACACAATGCATATCCACATAAATTAATATTTATTGTGCTCCTATTAATTCG } 17701845 \\ \text { DH Pahang chr11 TCGACACAATGCATATCCACATAAATTAATGTTTATTGTGCTCCTATTAATTCG } 22179706\end{array}$

Figure 3. Sequence alignment of 296bp fragment (M3) generated by primer Me2-Em1 with M. balbisiana (Pisang Klutuk Wulung, PKW) and M. acuminata (Double haploid Pahang) genome sequences based on banana-Hub BLAST tool. Highlighted letters show base mismatch and stars show difference from acuminata.
Me4-Em8 308bp CTTGTCCCAAGTAATAGGTTGGGCTAAGGTACAACGGGCTAGATAGAGAAGCGATGATGT 60 PKW chr2 CTTGTCCCAAGTAATAGGTTGGGCTGAGGTACAATAGGCTAGATAGAGGAGCGACGATGT $\mathbf{6 4 2 9 7 2 8}$ DH Pahang chr2 CTTGTCCCAAGTAATAGATTGGGCTGAGGTACAATGGGCTAGATAGAGGAGCGACGATGT 9983208

Figure 4. Sequence alignment of 308bp fragment (M4) generated by primer Me4-Em8 with M. balbisiana (Pisang Klutuk Wulung, PKW) and M. acuminata (Double haploid Pahang) genome sequences based on banana-Hub BLAST tool. Highlighted letters show base mismatch and stars show difference from acuminata. 


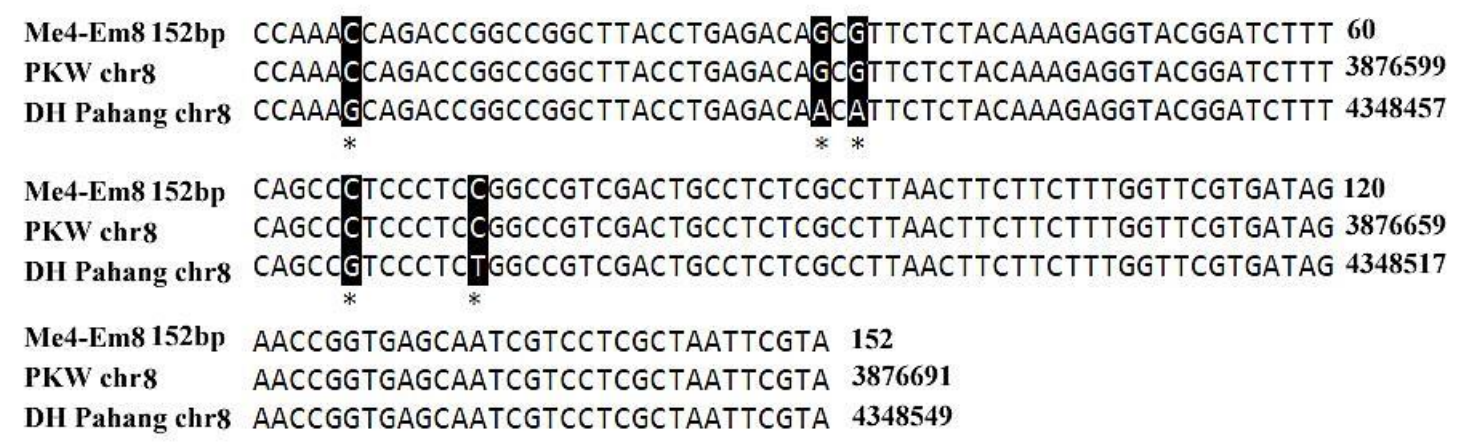

Figure 5. Sequence alignment of 152bp fragment (M5) generated by primer Me4-Em8 with M. balbisiana (Pisang Klutuk Wulung, PKW) and M. acuminata (Double haploid Pahang) genome sequences based on banana-Hub BLAST tool. Highlighted letters show base mismatch and stars show difference from acuminata.

For confirmation of sequence specificity, M3 and M5 sequences were used for specific primer design to amplify fragments of 246 and $256 \mathrm{bp}$ in size, respectively. Results showed that, the first reaction failed to generate specific fragment. However, the second reaction was successful in generating a specific band of the target size (256bp) which was amplified in all the B-genome contained accessions with exception of Butuhan, BB-545 and Pisang batu. Moreover, for more confirmation, four accessions of $M$. acuminata "AA" (i.e. Calcuta-4, Mal-399, Mal-250 and MalCICY) were introduced in this test and none of them was able to amplify the specific band (Fig. 6). These results indicate the efficiency of SRAP markers to expose minimum differences between genotypes. Genetic clarification of this kind of genetic variation could be elucidated by in situ hybridization of A and B pairing (Valdez-Ojeda et al. 2014). This may imply that chromosome re-assortments and exchanges of chromosome segments, leading to unbalanced genome transmission with respect to the parental (Jeridi et al. 2011). The presence and absence of some of the specific band for B genome in this study was similar to what Nair et al. (2005) have found using IRAP markers, thus they suggested mislabeling or misidentification.

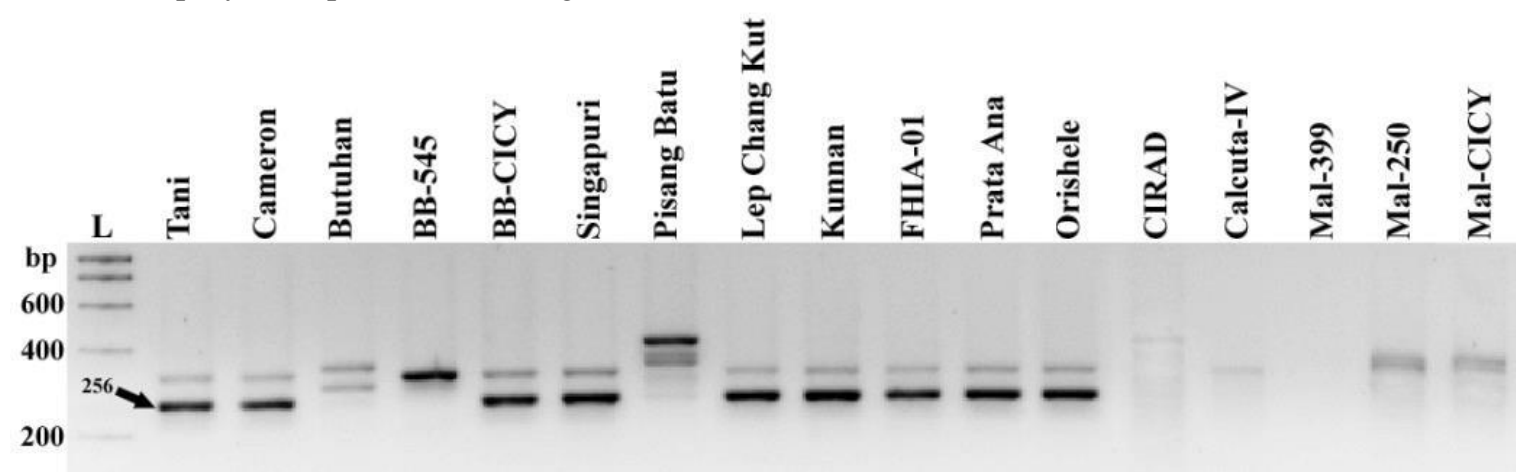

Figure 6. Amplification of a 256bp fragment (arrow) generated by specific designed primers, discriminating among $M$. balbisiana and M. acuminata related accessions.

In conclusion, molecular analysis performed in this study confirms the efficiency of SRAP markers in differentiation among $M$. balbisiana related accessions and indicates that SRAP is a suitable marker for genetic relationship and diversity assessment in Musa and other higher plants. The identification of the B genome specific bands generated by SRAP provided markers which could be used for identification of banana and plantain cultivars. This information is not only important in Musa basic breeding program but also in germplasm management and conservation as well as to authenticate mislabeled accessions.

\section{ACKNOWLEDGMENT}

This research work was funded by Secretaria de Agrícultura, Ganadería, Recursos Pesqueros y Alimentos (SAGARPA) and the Consejo Nacional de Ciencia y Tecnología (CONACyT) through the research grant 0048160 (RME). The first author (Dr. Youssef) is the recipient of a Postdoctoral fellowship from
CONACyT-TWAS (The World Academy of Science) award \# 3240267275. The authors acknowledge José Roberto Ku-Cauich for technical support.

\section{REFERENCES}

Ahmad, F.; R. Megia and Y.S. Poerba (2014) Genetic Diversity of Musa balbisiana Colla in Indonesia Based on AFLP Marker. HAYATI Journal of Biosciences, 21:(1) 39-47.

Crouch, J. H.; H. K. Crouch; H. Constandt; A. Van Gysel; P. Breyne M. Van Montagu; R.L. Jarret and R. Ortiz (1999). Comparison of PCR based molecular marker analyses of Musa breeding populations. Molecular Breeding, 5: 233-244.

D'Hont, A.; F. Denoeud; J.M. Aury et al. (2012) The banana (Musa acuminata) genome and the evolution of monocotyledonous plants. Nature, 488:213-21. 
Davey, M.W.; R. Gudimella; J.A. Harikrishna; L.W. Sin; N. Khalid and J. Keulemans (2013) A draft Musa balbisiana genome sequence for molecular genetics in ploidy, inter- and intra- specific Musa hybrids. BMC Genomics, 14:683. http://dx.doi.org/10.1186/1471-2164-14-683.

El-Khishin, D. A.; E.L. Belatus; A. Abd El-Hamid and K.H. Radwan (2009) Molecular charactarization of banana cultivars (Musa spp.) from Egypt using AFLP. Research Journal of Agriculture and Biological Sciences, 5: 272-279.

FAOSTAT (2015) Food and Agriculture Organization of the United Nations. http://faostat.fao.org.

Ferreira, C. F.; S.O. Silva; N.P.D. Sobrinho; S.C.S. Damascena; F.S.A.O. Alves and O. Da Pereira Paz (2004). Molecular characterization of banana (AA) diploids with contrasting levels of black and yellow sigatoka resistance. American Journal of Applied Sciences, 1: 276-278.

Häkkinen, M. (2013) Reappraisal of sectional taxonomy in Musa (Musaceae). Taxon, 62 (4): 809-813.

Hampl, V.; A. Pavlícek and J. Flegr (2001) Construction and bootstrap analysis of DNA fingerprintingbased phylogenetic trees with the freeware program FreeTree: application to trichomonad parasites. Int J Syst Evol Micr, 51:731-735.

Hari, P.C. (1989) Morphological study of Musa acuminata ssp. malaccensis (Ridley) Simmonds to determine taxonomic characteristics. Saussurea, 19:187-217.

Hippolyte, I.; C. Jenny; L. Gardes; F. Bakry; R. Rivallan; V. Pomies; P. Cubry; K. Tomekpe; A. M. Risterucci; N. Roux; M. Rouard; E. Arnaud; M. Kolesnikova-Allen and X. Perrier (2012) Foundation characteristics of edible Musa triploids revealed from allelic distribution of SSR markers. Ann Bot-London, 109:937-951.

Jaccard, P. (1908) Nouvelles recherches sur la distribution florale. Bull. Soc. Vaud. Sci. Nat., 44: 223-270.

Jeridi, M.; F. Bakry; J. Escoute; E. Fondi; F. Carreel; A. Ferchichi; A. D'Hont and M. Rodier-Goud (2011) Homoeologous chromosome pairing between the A and B genomes of Musa spp. revealed by genomic in situ hybridization. Ann Bot-London, 108:975-981.

Li, G. and C.F. Quiros (2001) Sequence-related amplified polymorphism (SRAP), a new marker system based on a simple PCR reaction: Its application to mapping and gene tagging in Brassica. Theoretical and Applied Genetics, 103: 455-461.

Nair, A.S.; C.H. Teo; T. Schwarzacher and P.H. Harrison (2005) Genome classification of banana cultivars from South India using IRAP markers. Euphytica, 144: 285-290.

Nei, M. (1987) Molecular Evolutionary Genetics. Columbia University Press, New York.
Nwakanma, D.C.; M. Pillay and B.E. Okoli (2003) PCR-RFLP of the ribosomal DNA internal transcribed spacers (ITS) provides markers for the A and B genomes in Musa L. Theor App Genet, 108:154-159.

Phothipan, S.; B. Silayoi; K. Wanichkul and S. Apisitwanich (2005) Genetic relationship among banana in $\mathrm{AA}, \mathrm{AAB}$ and $\mathrm{B}$ groups using random amplified polymorphic DNA (RAPD) and sequence related amplified polymorphism (SRAP) techniques. Kasetsart J. Nat. Sci. 39: 703-710.

Pinar, H.; A. Uzun; M. Unlu; M. Bircan; O. Gulsen; C. Yilmaz and H. Gubbuk (2015) Identification of Banana Accessions Sampled From Subtropical Region of Turkey Using SRAP Markers. Bulgarian Journal of Agricultural Science, 21 :(2) 270-276.

Poerba, Y. and A. Fajarudin (2010) Genetic variability among 18 cultivars of cooking bananas and plantains by RAPD and ISSR markers. Biodiversitas, 11:118-123.

Prevost, A. and M.J. Wilkinson (1999) A new system of comparing PCR primers applied to ISSR fingerprinting of potato cultivars. Theor Appl Genet, 98:107-112.

Risterucci, A. M.; I. Hippolyte; X. Perrier; L. Xia; V. Caig; M. Evers; E. Huttner; A. Kilian; J.C.Glaszmann (2009) Development and assessment of diversity arrays technology for high-throughput DNA analyses in Musa. Theoretical and Applied Genetics, 119: 10931103. doi: 10.1007/s00122-009-1111-5.

Roldan-Ruiz, I.; J. Dendauw; E. VanBockstaele; A. Depicker and M. De Loose (2000) AFLP markers reveal high polymorphic rates in ryegrasses (Lolium spp.). Mol Breed, 6: 125-134.

Shepherd, K. (1988) Observation on Musa taxonomy. In: Identification of genetic diversity in the genus Musa. Proc Int Workshop held at Los Banos, Philippines 5-10 September 1988. INIBAP, Montpellier, France, pp 158-165.

Sotto, R.C. and R.C. Rabara (2000) Morphological diversity of Musa balbisiana Colla in the Philippines. Info Musa, 9:28-30.

Stulnig, T.M. and A. Amberger (1994) Exposing contaminating phenol in nucleic acid preparations. BioTechniques, 16: 403-404.

Teo, C.H.; S.H. Tan; C.H. Ho; Q.Z. Faridah; Y.R. Othman; J.S. Heslop-Harrison; R. Kalendar and A.H. Schulman (2005) Genome constitution and classification using retrotransposon based markers in the orphan crop banana. J Plant Biol, 48: 96-105.

Till, B.; J. Jankowicz-Cieslak; L. Sági; O.A. Huynh; H. Utsushi; R. Swennen; R. Terauchi; C. Mba (2010) Discovery of nucleotide polymorphisms in the Musa gene pool by Ecotilling. Theor Appl Genet, 121:1381-1389. 
Ude, G.; M. Pillay; A. Nwakanma and A. Tenkouano (2002) Analysis of genetic diversity and selectional relationships in Musa using AFLP markers. Theor Appl Genet, 104: 1239-1245.

Valdez-Ojeda R.; A. James-Kay; J. Ku-Cauich and R.M. Escobedo-Gracia Medrano (2014) Genetic relationships among a collection of Musa germplasm by fluorescent-labeled SRAP. Tree Genetics and Genomes, 10: 465-476. DOI: 10.1007/s11295-013-0694-9.

Wang, X.; T. Chiang; N. Roux; G. Hao and X. Ge (2006) Genetic diversity of wild banana (Musa balbisiana Colla) in China as revealed by AFLP markers. Genet Resour Crop Evol, 54: 11251132.
Youssef, M. (2012) Khirshyat 1.0: a simple microprogram for some molecular biology protocols. Genes, genomes and genomics, 6:102-105.

Youssef, M.; A. James; R. Rivera-Madrid; R. Ortiz and R.M. Escobedo-Gracia Medrano (2011) Musa genetic diversity revealed by SRAP and AFLP. Mol Biotech, 47:189-199.

Youssef, M.; R. Valdez-Ojeda; J.R. Ku-Cauich and R.M. Escobedo-GraciaMedrano (2015) Enhanced Protocol for Isolation of Plant Genomic DNA. Journal of Agriculture and Environmental Sciences, $\quad 4:(2) \quad 172-180$. DOI: 10.15640/jaes.v4n2a20.

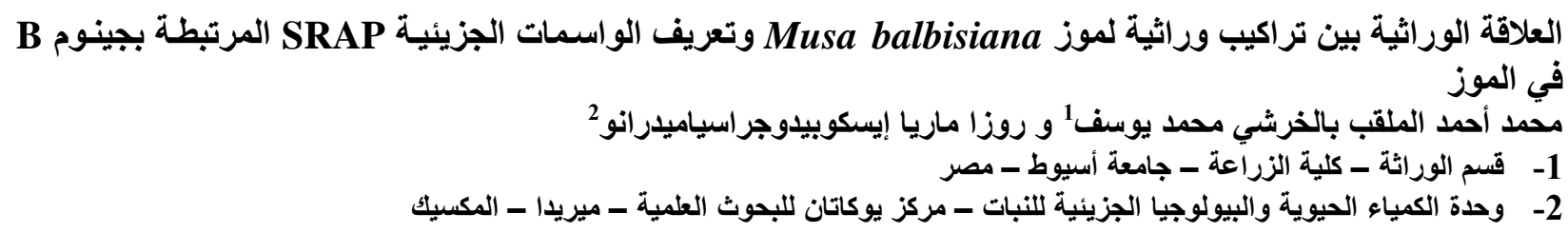

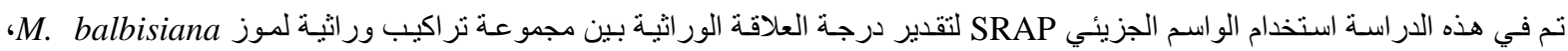

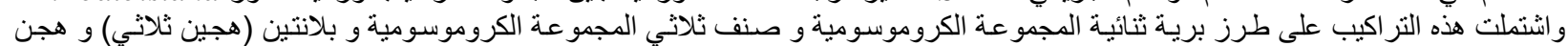

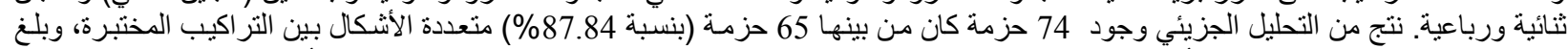

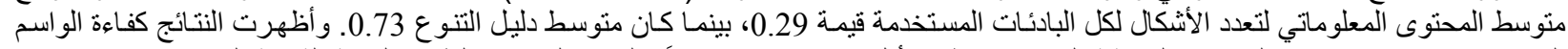
الجزيئي SRAP في تجميع التر اكيب الور اثية المختبرة وإظهار أقل فروق بينها.

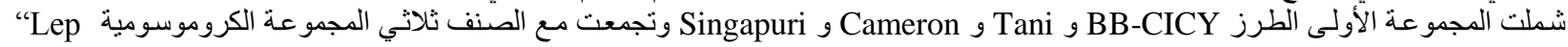

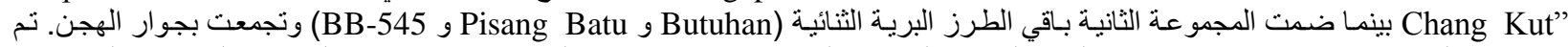

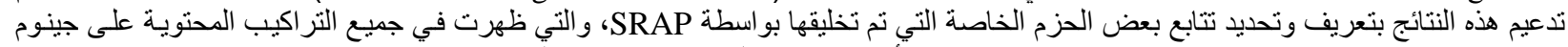

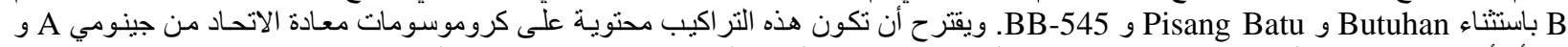

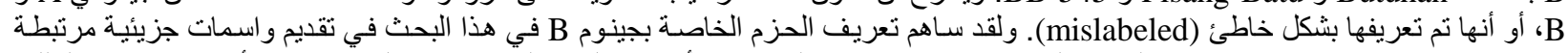

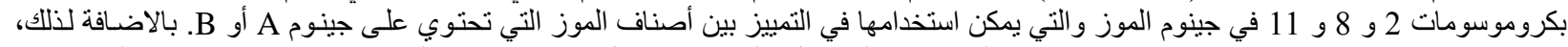

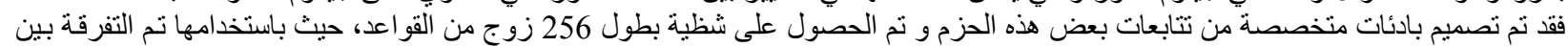

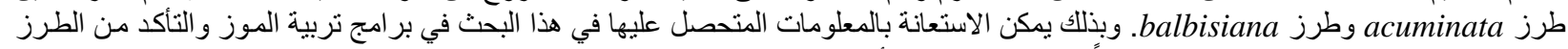

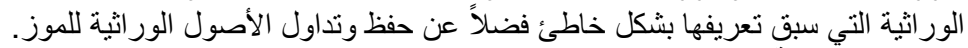

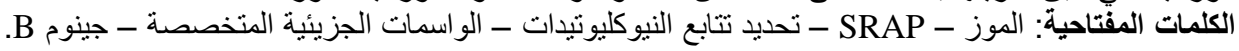

